\title{
Análise de Sensibilidade do Desenho de Roda no Coeficiente de Arrasto
}

\author{
Flavio Maruyama ${ }^{1}$, Felipe Magazoni ${ }^{1.2}$, Filipe Fabian Buscariolo ${ }^{1.3}$, Julio Cesar Lelis Passos ${ }^{1}$ \\ e Leonardo D. Volpe ${ }^{1}$ \\ ${ }^{1}$ GM Brasil \\ ${ }^{2} \mathrm{NETeF}-\mathrm{USP}$ \\ ${ }^{3} \mathrm{NDF}-\mathrm{USP}$
}

E-mails: felipe.magazoni@gm.com, filipe.buscariolo@gm.com, flavio.maruyama@gm.com, julio.alves@gm.com, leonardo.volpe@gm.com

\section{RESUMO}

Ano após anos, os fabricantes de automotores vem perseguindo cada vez mais a economia de combustível, ambos por pressões políticas e demanda da sociedade, o que leva as companhias a desenvolver novas soluções e tecnologias para melhorar a eficiência energética dos seus veículos.

Com advento dos softwares de CFD, é possível estudar propostas de redução do arrasto aerodinâmico, o que contribui para economia de combustível

Neste contexto, baseado em um modelo virtual de um sedan pequeno, correlacionado com teste em túnel de vento, foi montando 3 propostas de roda com raios em ângulo para verificar a influencia no coeficiente de arrasto.

Considerando que as rodas contribuem com $25 \%$ do total do arrasto de um veículo, este trabalho tem como objetivo apresentar a sensibilidade no coeficiente de arrasto com a mudança dos raios da roda para um sedan de pequeno porte. Os resultados encontrados apresentaram um decréscimo no desempenho aerodinâmico, contudo quando há o incremento do número de raios, observa-se um incremento no desempenho aerodinâmico devido ao fechamento da área aberta da roda.

\section{INTRODUÇÃO}

O projeto de novos carros buscam melhorar seu desempenho quanto a economia de combustível e a aerodinâmica é uma importante variável neste quesito. Para pequenos carros nas cidades, ser econômico tornou-se um objetivo, que pode ser alcançado com a combinação de redução de massa e o desenvolvimento da aerodinâmica sobre seu design e também na utilização de apêndices aerodinâmicos.

A roda representa uma das partes de maior interesse na aerodinâmica de um veículo, que pode ter diversas considerações, procurando desempenho aerodinâmico, refrigeração dos freios ou simplesmente estética. De acordo com HUCHO (1998) [6] e VDOVIN (2013) [9], 25\% de todo o arrasto de um veículo é devido as rodas, por esta razão que o estudo aerodinâmico de diversos tipos de desenhos de roda é objetivo central do estudo deste trabalho. A figura 1 descreve o impacto das variáveis envolvidas no consumo de combustível de um veículo comercial. 
Observe que a porção aerodinâmica corresponde $20 \%$ do total do consumo de combustível e os demais parâmetros são representados pelas condições climáticas, rota, mudança de velocidade, condutor e manutenção.

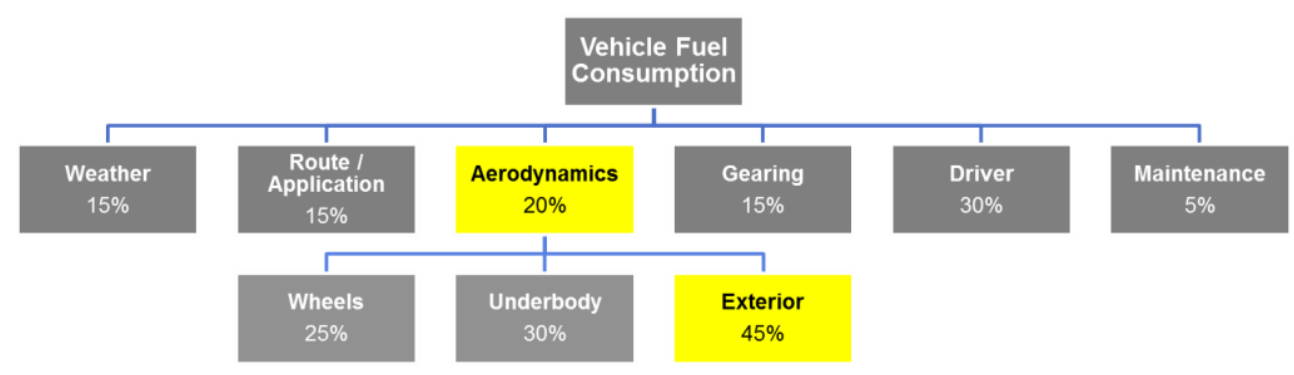

Figura 1. Consumo de combustível de um veículo comercial

Ao redor do mundo, há diversas iniciativas para regular as emissões e a eficiência de veículos, como a EURO para o mercado Europeu e INOVAR Auto para o mercado Brasileiro, que será implementada em 2017. A EURO determina limites de emissões para todos os tipos de veículos, no entanto, este trabalho irá considerar apenas veículos de passageiros. De acordo com a Agencia do Meio Ambiente Europeu (2014) [3], para veículos de passageiro, a regulamentação especifica $130 \mathrm{~g} \mathrm{CO} 2 / \mathrm{km}$ em 2015, como o valor médio para a frota recente de veículos de passageiros na UE. Para o período até 2015, cada modalidade tem um acordo de metas, que foram estabelecidas e são apresentados no capítulo seguinte. A meta de longo prazo é $95 \mathrm{~g}$ de CO2 / km e foi definido para 2021 (com aplicação gradual partir de 2020).

O projeto INOVAR Auto é um programa onde os fabricantes de veículos automotores precisam melhorar a eficiência média para novos VTL (veículos de trabalho leve) em 12\% dos níveis de 2012 para os veículos a serem lançados em outubro de 2017, para se qualificar para no programa. Este objetivo é baseado nas metas Europeias, onde novos VTL's tem que atingir 130 gCO $2 / \mathrm{km}$, e adaptado para o Brasil, baseado nas diferenças de ciclos de condução, veiculo, combustíveis e especificações de estradas. Para qualificar para uma redução adicional no IPI de $1 \%$ à $2 \%$, os fabricantes precisam atingir a média de eficiência dos veículos em outubro de 2016, que irá resultar em melhorias médias em novos veículos, cerca de $16 \%$ e 19\%, respectivamente.

Considerando o uso de CFD - Computational Fluid Dynamics, e na correlação com medições físicas de coeficiente de arrasto de diversos projetos, o delta $\mathrm{Cd}$ fornecido como resultado para este trabalho é confiável. Além, disso, a simulação virtual torna possível alterar facilmente as configurações e os desenhos de roda, o que pode ser muito complicado logisticamente e ter alto custo em um túnel de vento. Outro ponto a ser observado, é que no túnel de vento há muitas variáveis difíceis de serem isoladas, como a perda por arrasto dos pneus ou mesmo observar fenômenos específicos sem a interferência dos instrumentos de medição. Dentro do ambiente virtual, não há limites para estas observações.

Alguns trabalhos foram desenvolvidos para analisar a influência da abertura das rodas no coeficiente de arrasto aerodinâmico, medido em um veículo sedan de alto desempenho em condições estáticas e em movimento (Buscariolo e Karbon, 2011). O estudo foi conduzido usando simulações em CFD para avaliar os designs. 
Vdovin (2013) [9] tem como objetivo identificar os fatores no design de rodas que tem efeito significativo na resistência de ventilação para rodas com aro de 17 polegadas e o quanto isto afeta o desempenho aerodinâmico. Usando abordagem numérica, Vdovin (2014) [10] estudou a resistência de ventilação e os fatores que afetam seu comportamento.

Por muitos anos, muitos engenheiros e cientistas estudaram e estudam a aerodinâmica em rodas para tentar entender sua influência no arrasto. O objetivo principal deste trabalho é estudar a influência sobre o coeficiente de arrasto geral de um sedan de pequeno porte com rodas com diâmetro de 15 polegadas.

\section{FORMULAÇÃO MATEMÁTICA}

É necessário calcular as variáveis associadas ao escoamento baseada nas leis de conservação de massa, de momento e de energia, que é expressa através da expressão abaixo,

$$
\frac{\partial \rho \phi}{\partial t}+\operatorname{div}\left(\rho \phi u_{r}\right)=\operatorname{div}(\Gamma \operatorname{grad} \phi)+S_{\phi}
$$

onde $\rho$ é a massa específica do fluido, $t$ é o tempo, $u_{r}$ é a velocidade relativa entre o fluido e o sistema de coordenada, $\phi$ é uma variável escalar $(u, v, w, k, \varepsilon, E), \Gamma$ é o coeficiente de difusividade e $S_{\phi}$ é o termo fonte.

Substituem-se as variáveis escalares na Equação 1 e rearranjam-se a expressão, usando as equações de Navier-Stokes, que descrevem o movimento do fluido e suas propriedades, e considerando o escoamento incompressível, fluido Newtoniano isotérmico (massa específica e viscosidade são constantes), tem-se,

$$
\begin{gathered}
\nabla \cdot V=0 \\
\rho \frac{D V}{D t}=-\nabla P+\rho g+\mu \nabla^{2} V
\end{gathered}
$$

onde $P$ é a pressão, $\mu$ é a viscosidade dinâmica, $V$ é o campo de velocidade e $g$ é a aceleração da gravidade.

Como as equações de Navier-Stokes compõem um sistema aberto para ser computacionalmente resolvido, um modelo de turbulência é necessário para ser incorporado ao sistema. Neste trabalho, o modelo $k$ - $\varepsilon$ é considerado para resolver as equações de Navier-Stokes. O modelo de turbulência $k-\varepsilon$ é composto por duas equações que vem diretamente das formulações diferencias de transporte, em que $k$ representa a energia cinética turbulenta e $\varepsilon$ representa a taxa de dissipação turbulenta.

$$
\begin{gathered}
\frac{\partial \rho k}{\partial t}+\operatorname{div}\left(\rho k u_{r}\right)=\operatorname{div}\left(\frac{\mu_{t}}{\sigma_{k}} \operatorname{grad} k\right)+2 \mu_{t} E_{i j} E_{i j}-\rho \varepsilon \\
\frac{\partial \rho \varepsilon}{\partial t}+\operatorname{div}\left(\rho \varepsilon u_{r}\right)=\operatorname{div}\left(\frac{\mu_{t}}{\sigma_{\varepsilon}} \operatorname{grad} \varepsilon\right)+C_{1 z} \frac{\varepsilon}{k} 2 \mu_{t} E_{i j} E_{i j}-C_{2 z} \rho \frac{\varepsilon^{2}}{k}
\end{gathered}
$$


onde $\mu_{t}$ é a viscosidade turbulenta, $C_{1 z}, C_{2 z}, \sigma_{\varepsilon}$ e $\sigma_{k}$ são as constantes do modelo de turbulência.

Uma vez que o comportamento e as propriedades do fluido são conhecidos, o próximo passo é calcular o coeficiente de arrasto, que é função da pressão atuante no veículo devido ao contato com o ar. Sendo a força de arrasto (D) um resultado da solução das Equações de Navier-Stokes, o coeficiente de arrasto de um veículo, o qual é caracterizado pela sua área frontal $\mathrm{A}$ e velocidade $\mathrm{V}$, é expresso pela expressão abaixo,

$$
c_{D}=\frac{2 D}{\rho V^{2} A}
$$

onde $c_{d}$ é o coeficiente de arrasto, $\rho$ é a densidade do fluido e A é a área projetada.

Para o Resistencia de ventilação, o coeficiente pode ser definido utilizando a mesma equação utilizada anteriormente, Vdovin (2013),

$$
c_{d(\text { vent })}=\frac{2 F_{\text {trac }}}{\rho V^{2} A}
$$

onde $c_{d(v e n t)}$ é o coeficiente de resistência à ventilação e $F_{t r a c}$ é a força correspondente para resistência e ventilação.

A equação 7 pode ser usada quando há necessidade de entender o efeito da resistência e ventilação e seus impactos no desempenho aerodinâmico total. Neste estudo, como simplificação, apenas o coeficiente aerodinâmico total será avaliado.

\section{DETALHES DO TÚNEL DE VENTO}

Para realizar o estudo de simulação CFD, o túnel de vento da General Motors (GM), um dos maiores túneis do mundo, foi considerado. O túnel consiste de um sistema de circuito fechado com controle de temperatura utilizado para realizar os estudos aerodinâmicos tais como estabilidade direcional e conforto térmico em diversos tipos de veículos. A seção de teste possui piso estático com um sistema de sucção da camada limite no início da seção.

De acordo com KELLY et al. (1982) [11], o túnel da GM apresenta uma área de seção de 56.16 $\mathrm{m}^{2}$ e um comprimento de aproximadamente $21.3 \mathrm{~m}$. É construído de metal revestido por uma camada de concreto de $600 \mathrm{~mm}$ com o objetivo de prevenir a propagação de som. O escoamento de ar é realizado por um poderoso ventilador de $13 \mathrm{~m}$ de diâmetro e uma potência de aproximadamente $3000 \mathrm{~kW}$, capaz de alcançar velocidades de até $250 \mathrm{~km} / \mathrm{h}$ por 15 minutos. Um esquema do túnel de vento da GM é apresentado na Figura 3.

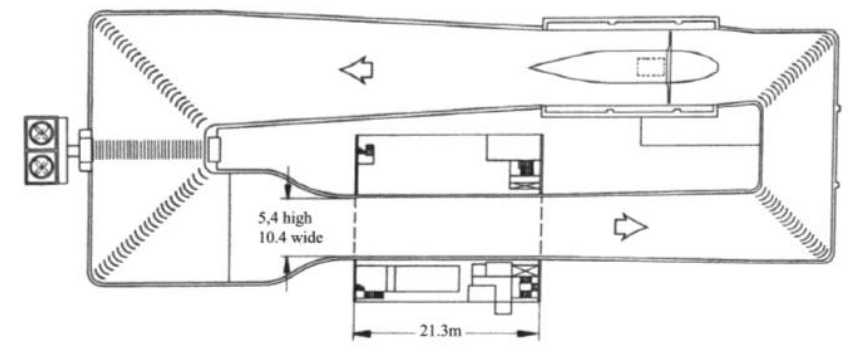

Figura 3. Túnel de vendo da General Motors (Hucho, 1998) [10] 
O túnel de vento da GM possui piso estático combinado com um sistema de sucção de camada limite para reduzir seu efeito sobre o coeficiente de arrasto do carro. Muitos estudos vêm sendo realizados para entender o comportamento do sistema de sucção de camada limite no túnel de vento e como evitar sua interferência nas medições aerodinâmicas. Neste modelo, não existe rotação das rodas, que podem interferir no escoamento ao redor do veículo.

O método que melhor aproxima às condições reais de um veículo é aplicando esteira rolante no piso da seção de teste do túnel de vento. A ideia é reproduzir a condição de uma estrada, onde o veículo está se locomovendo contra o ar. Para que tal condição ocorra no túnel de vento, a esteira deve se movimentar com a mesma velocidade do ar. As rodas do veículo devem também se movimentar e estar em contato com a esteira. Neste presente trabalho, esta aproximação será utilizada no desenvolvimento das simulações.

\section{METODOLOGIA}

Neste Trabalho, a metodologia proposta é baseada na comparação do coeficiente de arrasto de diferentes propostas de configurações de rodas em um sedan de produção utilizando CFD. A simulação considera o solo em movimento e a rodas em rotação, montados em um túnel de vento virtual. O túnel de vento para este trabalho possui dimensões similares aos do túnel da General Motors (GM), localizado em Warren, Estados Unidos.

Como no trabalho de BUSCARIOLO e KARBON (2011) [2], são utilizados parâmetros similares de simulação, expressos na tabela abaixo:

Tabela 1. Parâmetros da simulação computacional

\begin{tabular}{|l|c|}
\hline Túnel de vento & General Motors, Warren-Michigan \\
\hline Velocidade do ar & $110 \mathrm{~km} / \mathrm{h}(30.56 \mathrm{~m} / \mathrm{s})$ \\
\hline Pressão de saída & Pressão atmosférica \\
\hline Intensidade da turbulência & $0.60 \%$ \\
\hline Sistema de sucção da camada limite & No início da seção de teste \\
\hline Dimensões da seção do teste & $(5.4 \times 10.4 \times 23.0) \mathrm{m}$ \\
\hline Modelo de turbulência & $k-\varepsilon$ \\
\hline
\end{tabular}

As simulações foram realizadas utilizando o software FLUENT®. Hypermesh® foi utilizado geração da malha $2 \mathrm{D}$, no veículo e no túnel; T-Grid ${ }^{\circledR}$ foi utilizado para geração da malha volumétrica. O veículo em estudo é um sedan, representado por um modelo virtual, considerando o motor e o sistema de arrefecimento. O modelo do veículo proposto e os modelos de roda propostos podem ser vistos na Figura 4.

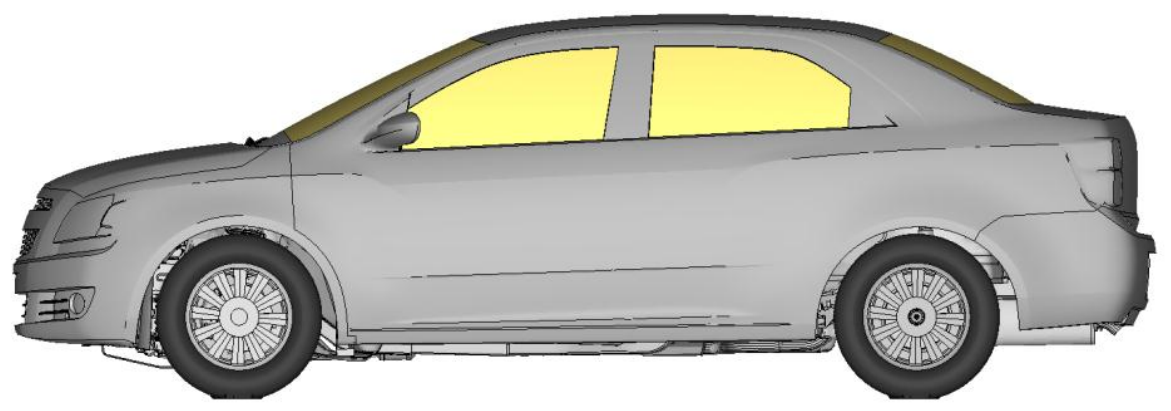

Figura 4. Representação do veículo estudado neste trabalho 
A figura 5 representa cada proposta de roda avaliada. $\mathrm{O}$ modelo de referência, considera o raio plano e as propostas consideram, da parte superior até a inferior, como ângulo positivo, o segundo como negativo e o terceiro sendo uma combinação dos dois anteriores. A esteira lateral para o modelo de referência, proposta 1, 2 e 3 apresentaram perfil similar. A proposta 4 tem um ponto de alta energia na esteira traseira, o que energiza o escoamento e leva a um menor coeficiente de arrasto.

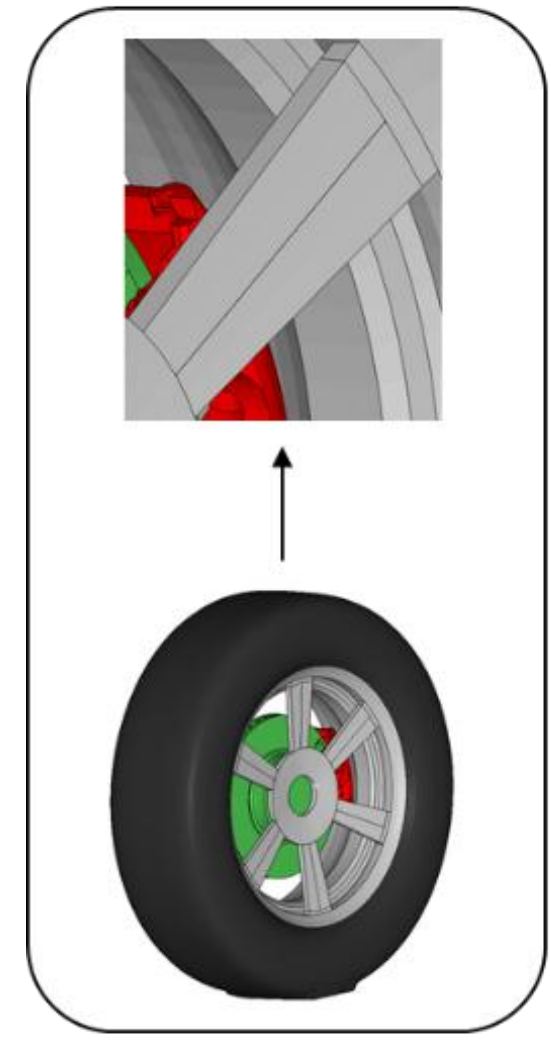

BASELINE DESIGN

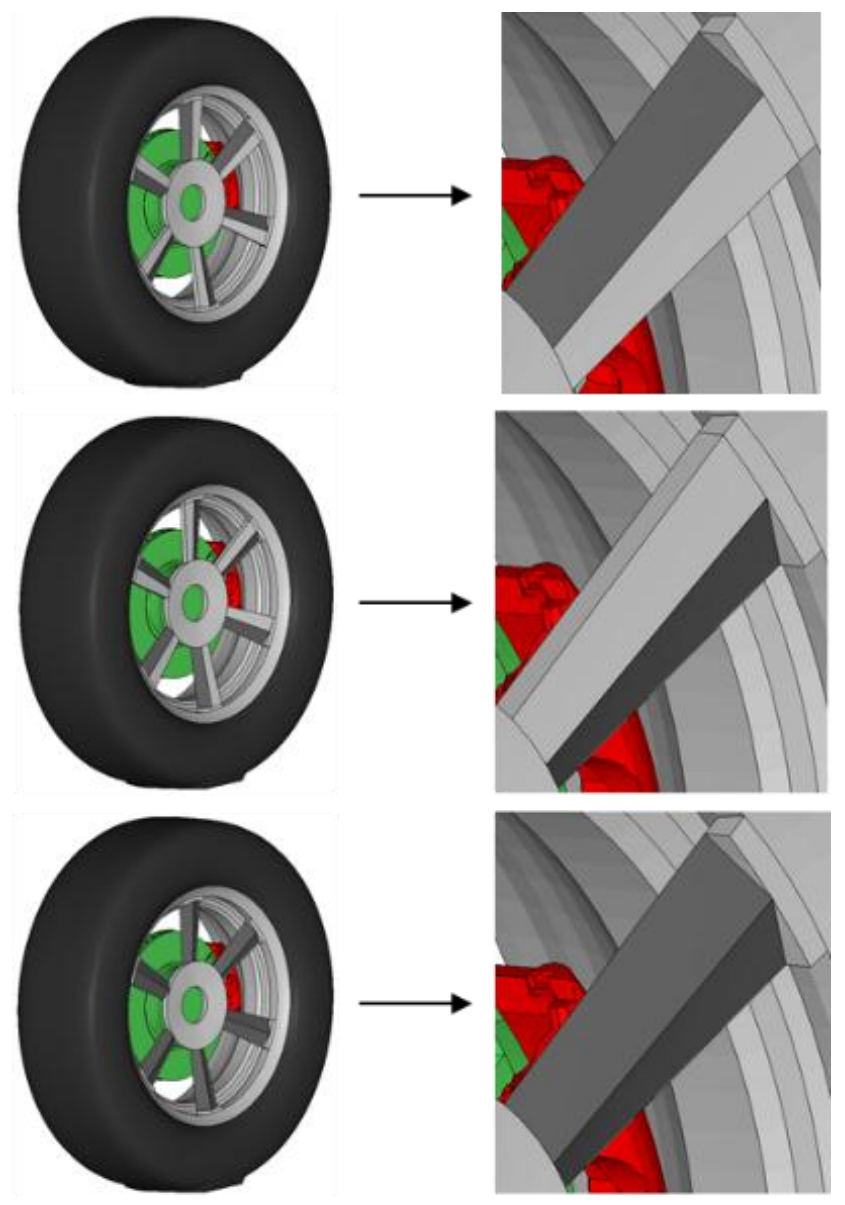

PROPOSED WHEEL DESIGNS

Figura 5. Representação do modelo de referência e das 3 propostas

\section{RESULTADOS}

Comparando os resultados entre o modelo de referência e as outras 3 propostas são sumarizados na tabela 2 o e melhor resultado foi obtido pelo modelo de referência.

Assim, adiciona-se entre as outras 3 propostas, uma quarta que é a modificação do melhor conceito, adicionando mais raios para compreender o comportamento desta modificação com relação ao fechamento da abertura da roda, como pode ser visto na figura 6 . 


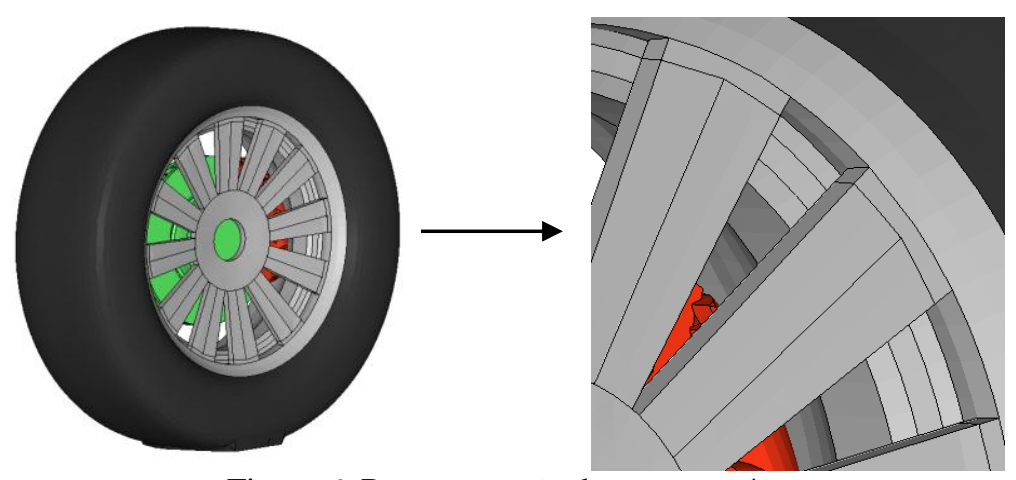

Figura 6. Representação da proposta 4

Considerando o arrasto total avaliado, é possível observar que a quarta proposta tem o melhor desempenho, com redução de 0.007 comparado com o modelo de referência. Os resultados podem ser vistos abaixo, na tabela 2 .

Tabela 2. Tabela com resultados do coeficiente de arrasto

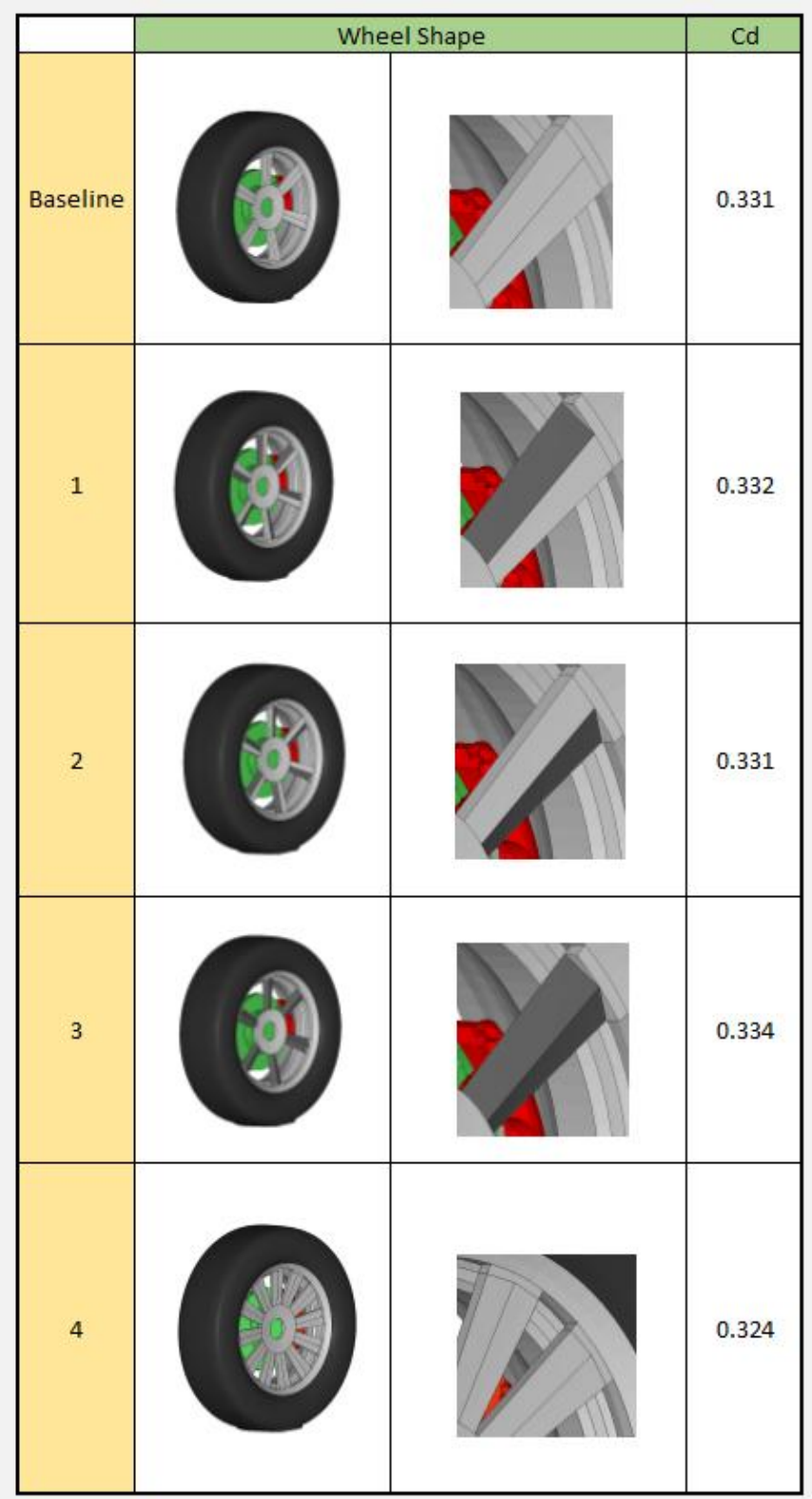




\section{CONCLUSÃO}

A figura 7 mostra a comparação dos perfis da esteira na vista lateral do veículo.
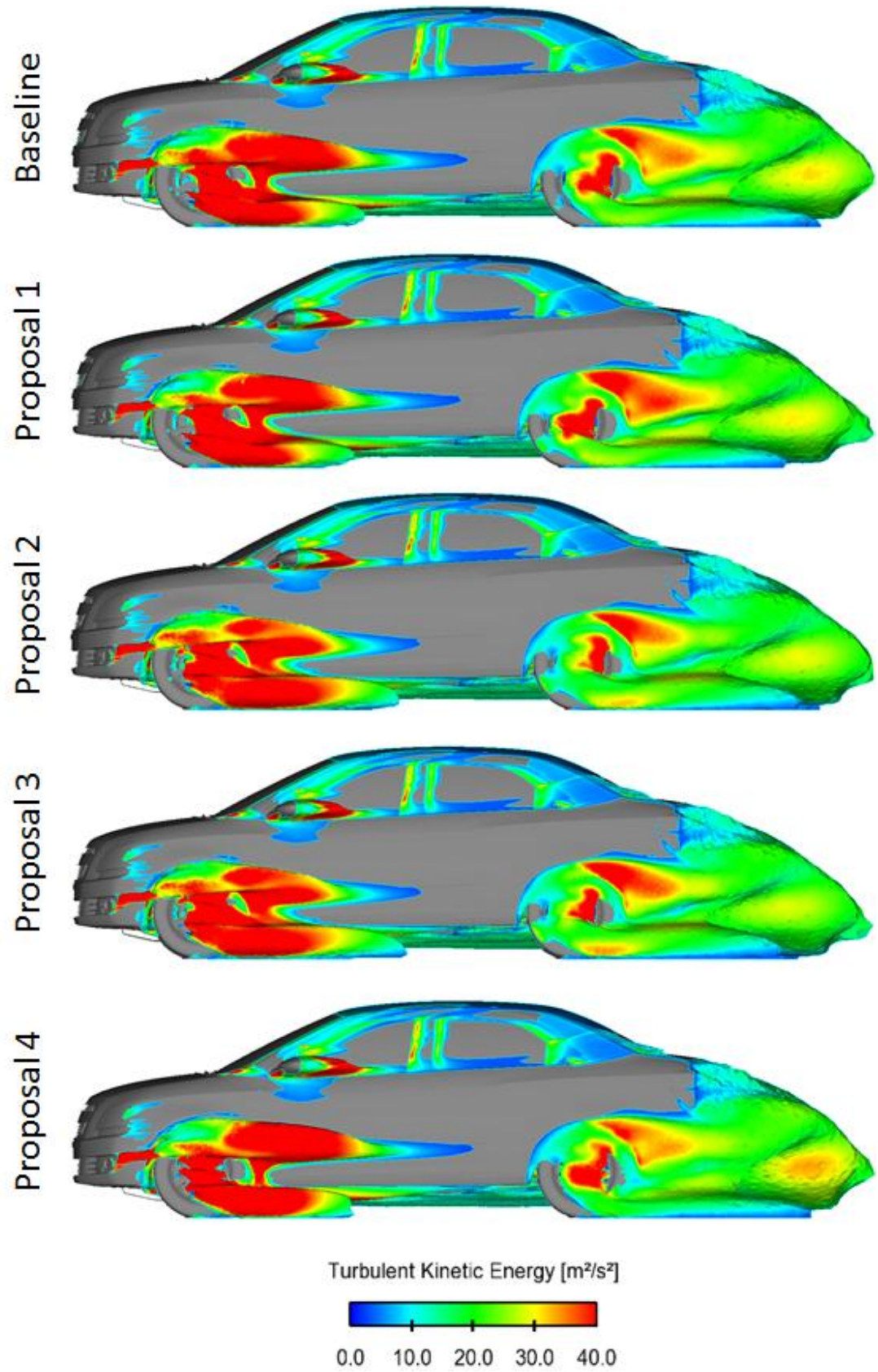

Figura 7. Comparativo das de esteira colorida por energia cinética turbulenta - vista lateral

A vista superior das esteiras é apresentada na figura 8 e todas apresentam perfil similar, uma vez que a principal mudança ocorre na roda, desenho, nenhuma mudança significativa e observada nesta vista.

A proposta 4 apresenta melhor resultado comparado ao modelo de referência, no qual é baseada. Mostrando que a redução na abertura da roda pode melhorar o desempenho aerodinâmico. Este resultado é visível na esteira produzida, e pode ser verificado nos trabalhos BUSCARIOLO e KARBON (2011), VDOVIN (2013) e VDOVIN (2014). Embora os carros e o tamanho das 
rodas sejam diferentes, o trabalho apresenta uma tendência similar de melhora no coeficiente de arrasto com o fechamento da roda. Neste trabalho, a redução encontrada foi de 0.007 .
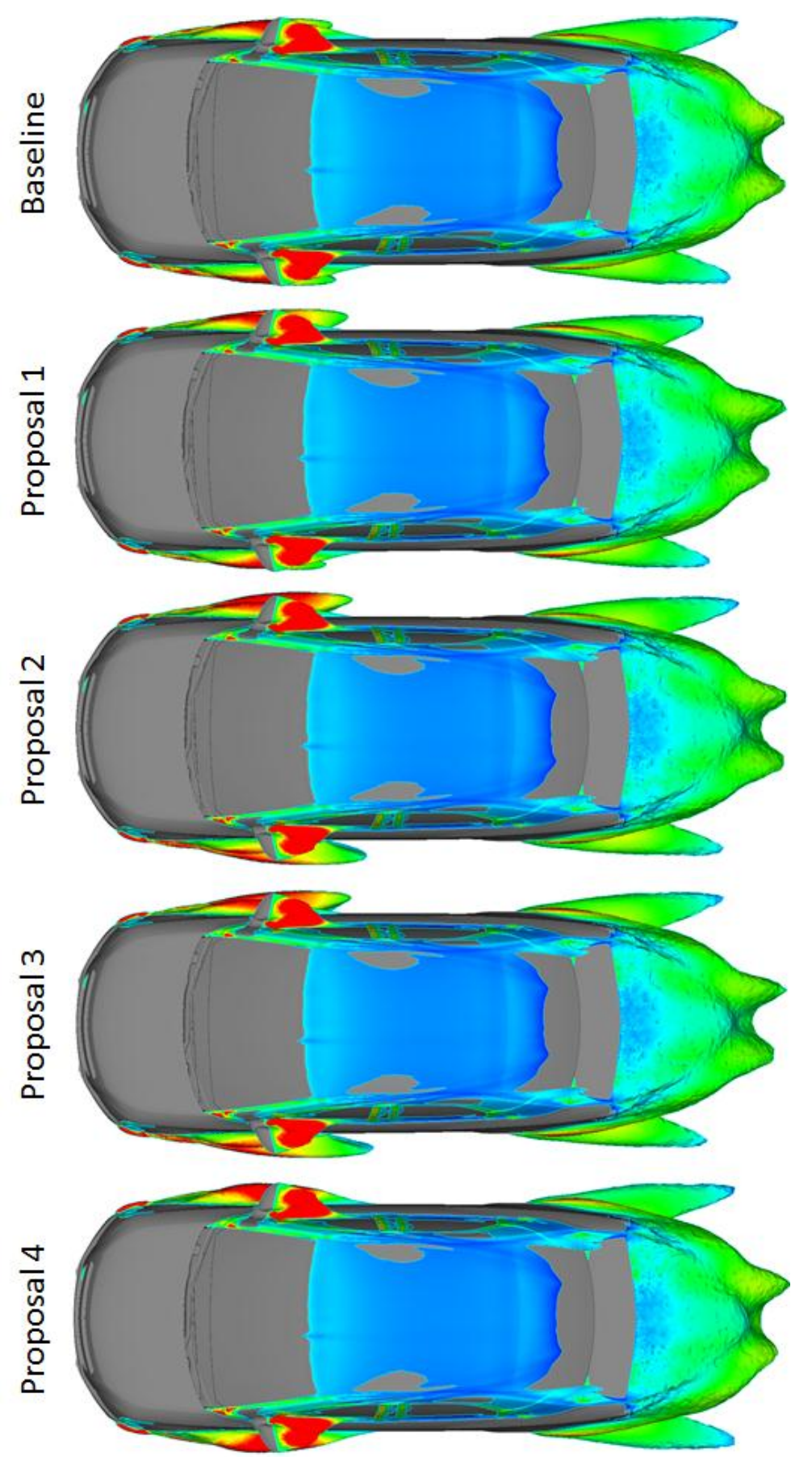

Turbulent Kinetic Energy $\left[\mathrm{m}^{2} / \mathrm{s}^{2}\right]$

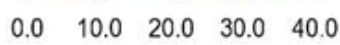

Figura 8. Comparativo das de esteira colorida por energia cinética turbulenta - vista superior 


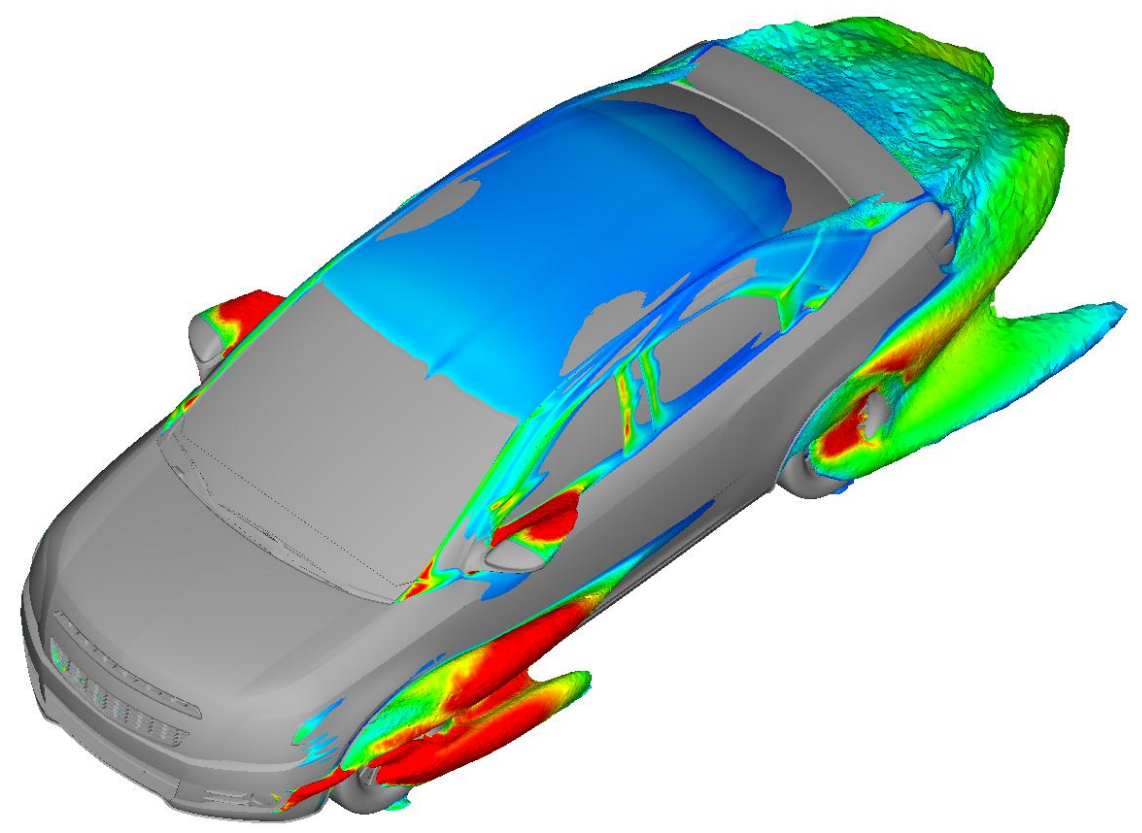

Figura 9. Vista isométrica da esteira da proposta 4.

Como consideração final, é possível verificar que os raios podem influenciar no coeficiente de arrasto total e que isto deve ser levado em conta no desenvolvimento de uma nova roda, incluindo o número de raios que interferem no escoamento do ar. Outro ponto que deve ser observado é a porção que diz respeito ao resfriamento dos freios, que não foi avaliado neste estudo.

\section{NOMENCLATURA}

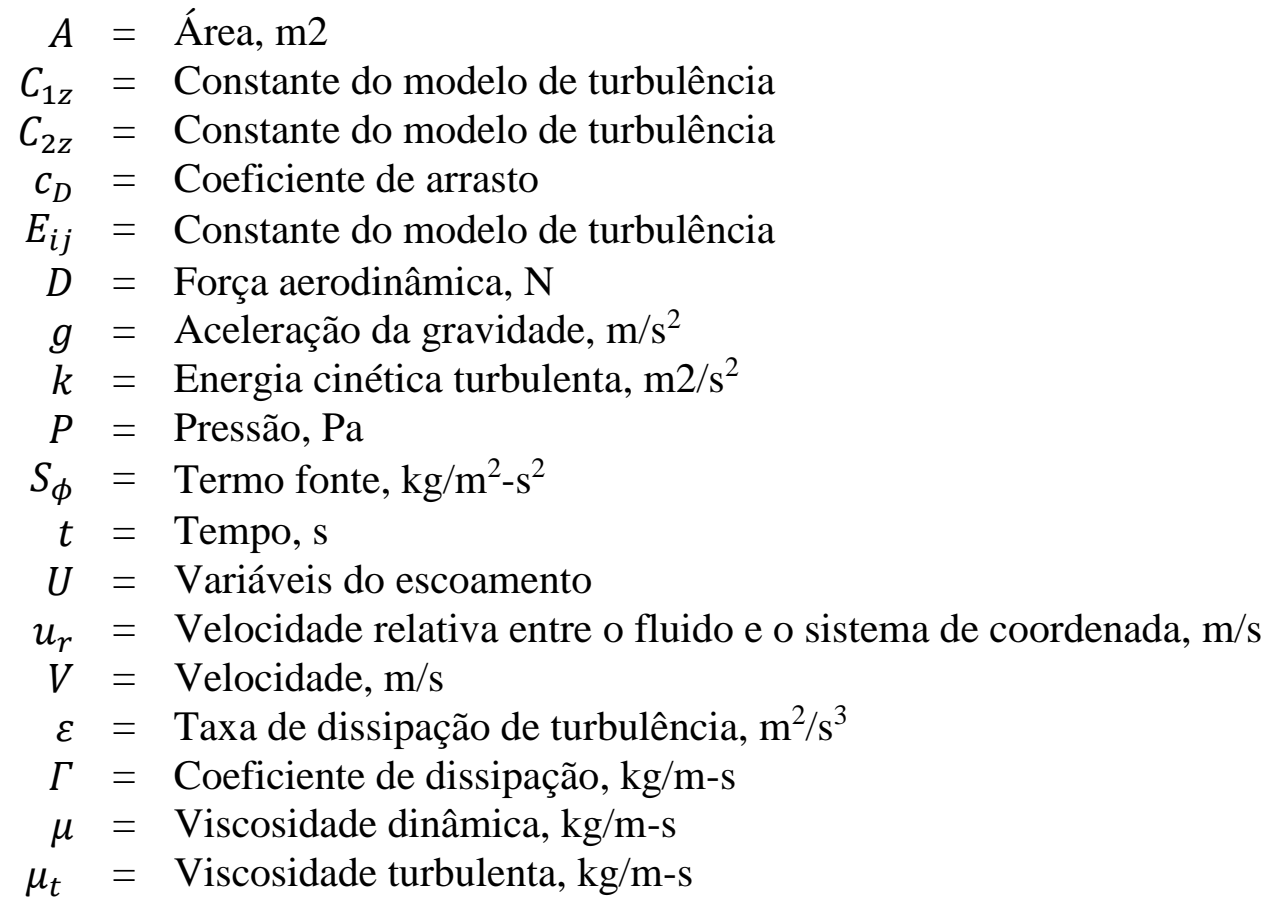


$\phi=$ Variável escalar

$\rho=$ Massa específica, $\mathrm{kg} / \mathrm{m}^{3}$

$\sigma_{\varepsilon}=$ Constante do modelo de turbulência

$\sigma_{k}=$ Constante do modelo de turbulência

\section{REFERÊNCIAS}

1. BUSCARIOLO, F.F., Analysis of Different Types of Wind Tunnel's Ground Configuration Using Numerical Simulation, SAE Paper, N ${ }^{\circ}$ 2010-36-0078I, Society of Automotive Engineers, 2010.

2. BUSCARIOLO, F.F.; KARBON, K.J., Comparative CFD Analysis Between Rotating and Static Cases of Different Wheels Opening Designs over a Performance Sedan, SAE Paper, No 2011-36-0271, Society of Automotive Engineers, 2011.

3. European Environment Agency, Monitoring $\mathrm{CO} 2$ emissions from passenger cars and vans in 2013, EEA Technical report No 19/2014, 2014.

4. FLUENT 14.0., User's Guide, Fluent Inc., 2011.

5. Hypermesh 11.0 User's manual Altair Computing, Inc., 2011.

6. HUCHO, W. H., Aerodynamics of Road Vehicles, From Fluid Mechanics of Vehicle Engineering, 4th Edition, SAE International, 1998.

7. KELly, K. B.; PROVENCHER, L. G.; SCHENKEL, F. K., The General Motors Engineering Staff Aerodynamics Laboratory - A full Scale Automotive Wind Tunnel, SAE Paper, No 820371, Society of Automotive Engineers, 1982.

8. Lofdahl, L.; Sebben, S., Investigation of Wheel Aerodynamic Resistence of Passenger Cars, SAE Paper, 2014-01-0606, Society of Automotive Engineers, 2014.

9. Vdovin, A., Investigation of Aerodynamic Resistence of Rotating Wheels on Passenger Cars, Thesis for the degree of Licentiate of Engineering, Chalmers university of Technology, Sweden, 2013.

10. Vdovin, A.; Lodfdahl, L., Investigation of Wheel Aerodynamic Resistance of Passenger Cars, SAE Paper, Society of Automotive Engineers, 2014.

11. Wäschle. AJ., "The Influence of Rotation Wheel on Vehicle Aerodynamics - Numerical and Experimental Investigations", SAE-Paper 2007-01-0107, 2007

12. WHITE, F., Fluid Mechanics - fouth edition, McGraw Hill, 1999

13. WIEDEMANN, J., The Influence of Ground Simulation and Wheel Rotation on Aerodynamic Drag Optimization - Potential for Reducing Fuel Consumption, SAE Paper, No 960672, Society of Automotive Engineers, 1996. 